\title{
LINEAMIENTOS PARA LA CÁTEDRA DE LA PAZ EN LA FUNDACIÓN INSTITUTO CRISTIANO DE PROMOCIÓN CAMPESINA (ICPROC), A PARTIR DEL PENSAMIENTO DE CAMLO TORRES RESTREPO
}

Rubén Alfredo Carrillo Carrillo. 


\section{GUIDELINES FOR THE CATHEDRA OF PEACE IN THE INSTITUTO CRISTIANO DE PROMOCIÓN CAMPESINA (ICPROC) FOUNDATION, FROM THE THOUGHT OF CAMILO TORRES RESTREPO.}

\begin{abstract}
The promulgation of the law 1732 of 2014 and the decree 1038 of 2015, implanting the cathedra of peace in all educational establishments, created the necessity that, institutions such as the ICPROC Foundation, to review their study plan to add this new subject. With the objective of establish the guidelines for the cathedra of peace in the ICPROC Foundation from the thought of Camilo Torres Restrepo. The ideas exposed previously, conduced us to ask ourselves: How can we use the thought of Camilo Torres Restrepo to the cathedra of peace in ICPROC Foundation?" To answer the question, it has been made a qualitative analysis, addressed from three different points of view. On the one hand, the ICPROC Foundation, for which it was necessary the interview analysis, which allowed us to know the opinion of pedagogical advisors and teacherstutors of the ICPROC Foundation; next to the analysis of the PEI's document, of the same Foundation. On the other hand, the semiotic analysis of the speech, that helped us to extract the bases of the thought of Camilo, in four of his writings, all with a reference to education. Finally, in the light of the document analysis, the law 1732 of 2014 and decree 1038 of 2015 was examined.
\end{abstract}

Key words: Cathedra of peace, Education for peace, Education about peace, Education in peace, Camilo Torres Restrepo, ICPROC Foundation, peace.

\section{AUTOR}

Rubén Alfredo Carrillo Carrillo.

Sacerdote de la Diócesis de Barrancabermeja. Licenciado en Filosofía y Educación Religiosa Universidad Católica del Oriente (UCO).

Correo electrónico: rcarrillo14@unab.edu.co

\section{RESUMEN}

La promulgación en Colombia de la Ley 1732 de 2014 y del Decreto 1038 de 2015 que implanta la Cátedra de la Paz en todos los establecimientos educativos del país, crea la necesidad de revisar el plan de estudios en instituciones como la Fundación ICPROC, para incluir la nueva área. La investigación tiene como objetivo establecer los lineamientos para la Cátedra de la Paz en la Fundación Instituto Cristiano de Promoción Campesina (ICPROC), a partir del pensamiento de Camilo Torres Restrepo, como fundamento de una Educación para la Paz. Lo expuesto anteriormente, nos condujo a preguntarnos: “¿Cómo aplicar el pensamiento de Camilo Torres Restrepo a la Cátedra de Paz en la Fundación Instituto Cristiano de Promoción Campesina (ICPROC)?". Para responder esta interrogante se realizó una investigación cualitativa, abordada desde tres puntos de vista distintos: análisis de entrevistas en la Fundación ICPROC; análisis del PEI de esta Fundación; y análisis semió tico del discurso en las bases del pensamiento de Camilo, en cuatro de sus escritos, todos con alguna referencia a la Educación. Finalmente, a la luz del análisis de documento, se examinó la Ley 1732 de 2014 y el Decreto 1038 de 20015.

Palabras clave: Cátedra para la Paz, Educación para la Paz, Educación sobre Paz, Educación en Paz, Camilo Torres Restrepo, Fundación ICPROC, paz. 


\section{INTRODUCCION}

Colombia atraviesa por un momento determinante en su historia. La firma de los acuerdos de paz con la guerrilla de las FARC (BBC Mundo, 2016) y el inicio de los diálogos con el ELN (BBC Mundo, 2017), auguran vientos nuevos en la construcción de la Patria. Ya el gobierno nacional se ha venido preparando para esta nueva realidad, es así como el Congreso de la República estableció la Cátedra de la Paz en todas las instituciones educativas del país, por medio de la Ley 1732 del 1 de septiembre de 2014. Esta Ley, fue reglamentada por el Presidente de la República, en el Decreto 1038 del 25 de mayo de 2015, determinando la incorporación de "la asignatura de la Cátedra de la Paz dentro del Plan de Estudios antes del 31 de diciembre de 2015" (artículo 3). Pero se descubre en la mayoría de las instituciones educativas del país, un vacío de procedencia, pues aún los docentes y directivos no encuentran el camino para hacer de la Cátedra de la Paz una realidad dentro de los establecimientos educativos (Charria, 2016). Situación que se presenta instituciones de educación formal, no formal e informal, como la Fundación Instituto Cristiano de Promoción Campesina (ICPROC).

Frente a la realidad expuesta en el párrafo precedente, se buscó la manera de ayudar a la Fundación ICPROC en la integración de la Cátedra de la Paz dentro de su Plan de Estudios. Muchas eran las posibilidades e ideologías que se podían tener, el investigador se inclinó por el pensamiento de Camilo Torres Restrepo, pues nadie mejor que un alzado en armas, para ayudarnos en la construcción de la nueva Patria, sin el deseo de empuñarlas; para descubrir que la revolución ya no es con la guerra, sino con las ideas. Además se hace pertinente, al cumplir los 50 años de la muerte de Camilo,
Que su pensamiento social y teológico, su liderazgo por la unidad, su obra y sus cenizas, vuelvan a la memoria del pueblo y de la Iglesia, al patrimonio acumulado para la paz con verdad y justicia social, con reconciliación y perdón como piso de garantías del NUNCA MÁS colombiano (Monsalve, 2015, p. 8).

Pues Camilo, como lo afirmó el Padre Javier Giraldo, en su discurso de apertura del Acto Ecuménico en Memoria de Camilo, el 7 de noviembre de 2015, en Cali: "murió fuertemente estigmatizado, diríamos: arrojado a los dominios del mal".

Lo anteriormente expuesto nos condujo a preguntarnos: ¿Cómo aplicar el pensamiento de Camilo Torres Restrepo a la Cátedra de la Paz en la Fundación Instituto Cristiano de Promoción Campesina (ICPROC)? Para responder al interrogante planteado, se propuso la presente investigación, con el objetivo de establecer los lineamientos para la Cátedra de la Paz en la Fundación Instituto Cristiano de Promoción Campesina (ICPROC), a partir del pensamiento de Camilo Torres Restrepo, como fundamento de una educación para la paz. Para alcanzar el objetivo general, fue necesario dar los siguientes pasos: 1. Realizar un diagnóstico sobre la educación para la paz en la Fundación Instituto Cristiano de Promoción Campesina (ICPROC); 2. Estudiar las directrices del Ministerio de Educación Nacional sobre la Cátedra de la Paz; 3. Identificar los elementos pedagógicos en el pensamiento de Camilo Torres Restrepo para el fomento de una educación para la paz; por último, 4. Proponer los temas, perspectivas y metodologías de la Cátedra de la Paz para la Fundación Instituto Cristiano de Promoción Campesina (ICPROC). 
Este trabajo investigativo es de gran actualidad tanto para la sociedad colombiana como para el presente educativo de la Fundación Instituto Cristiano de Promoción Campesina (ICPROC). En cuanto a la sociedad, porque responde a un deseo connatural del ser humano, alcanzar la paz; por tanto es válido hacer una propuesta de reconciliación desde el pensamiento de un revolucionario (Camilo Torres Restrepo). En la educación de la Fundación ICPROC, porque le permite institucionalmente pensar dentro de su plan de estudios, la Cátedra de la Paz, para responder a las directrices del Ministerio de Educación Nacional y además, empezar a construir desde el objetivo mismo de la institución, la promoción integral de los campesinos, que haga de cada uno de ellos, líderes en sus comunidades, de procesos de convivencia.

\section{EDUCAR PARA LA PAZ}

En torno a la Educación para la Paz encontramos a José Luis Zurbano (1998), quien afirma que educación para la paz es educar para la vida, para solucionar los problemas cotidianos; por lo cual, es necesario formar en valores, dentro de un clima de paz. Este autor considera además que la educación para la paz debe ser un proyecto transversal en tres niveles: intelectual, afectivo y conductual. Por su parte, Benito Olleros González (2000) presenta la importancia de una escuela donde la educación sea significativa y humana, donde se promueva la solidaridad, cooperación, autoestima y creatividad, de donde surge el proyecto transversal de educación para la paz.

En una recopilación realizada por Oswald (2001) se resaltan dos investigaciones: la primera realizada por Ada Ahorani quien pone de relieve la importancia de la cultura, la literatura y el arte en la transformación de la realidad; la segunda realizada por Sepideh Rouahani, quien retomando los escritos Baha'i, manifiesta la importancia de la dimensión espiritual, unida a la dimensión material y que lo único que pretende es llegar a una nueva raza de hombres y mujeres. Además, García y López (2011) presentan como competencias que se deben desarrollar en la escuela el saber participar, saber cooperar, ser responsables, tolerantes, solidarios, disponer de habilidades sociales y aprender a gestionar pacíficamente los conflictos interpersonales. Finalmente, Bautista (2012) presenta el conflicto yla agresividad como parte de la naturaleza humana; además,distingue cuatro clases de violencia: directa (causar daño a otro), estructural (ejercida por el medio), cultural (la cultura acepta la violencia) y simbólica (dominación).

En América Latina se destacan tres investigaciones. La primera, realizada por Hirmas y Carranza (2008), quienes consideran que la educación para la paz debe ser transversal desde las competencias (aprender a ser, aprender a convivir, aprender a conocer y aprender a hacer), el enfoque (formación en valores, educación para la vida y ejercicio de derechos humanos) y la metodología (centrada en el estudiante, trabajo en equipo, aprendizaje significativo y cooperativo). La segunda investigación es de Salazar y Sandoval (2009), quienes desarrollan ideas en torno a la finalidad de educación para la paz: formar, informar y transformar, desde la educación en valores $\mathrm{y}$, la importancia de la claridad del currículo (qué), los objetivos (para qué) y el método (cómo). La tercera investigación es elaborada por Gómez (2012) quien considera que la educación para la paz debe ser 
transversal y centrada en los valores; además hace un paralelo entre paz negativa (ausencia de violencia directa), paz positiva (ausencia de violencia indirecta o estructural) y paz neutra (ausencia de violencia cultural y simbólica).

En Colombia se desarrollan esencialmente tres ideas: la educación para la paz es la oportunidad para impulsar y favorecer todas las capacidades de las personas, disminuyendo la pobreza, la opresión y la alienación (Salamanca A. P., 2009). La educación para la paz se desarrolla en un trabajo conjunto de todos los miembros de la comunidad educativa y los entes gubernamentales (Jiménez, Lleras \& Nieto, 2010). Los jóvenes consideran que el conflicto es ajeno a ellos, puesto que en su pensamiento el conflicto solo se presenta a gran escala (la guerra); se considera que el juego y la cooperación ayudan a la cultura de paz, el primero estrecha relaciones, el segundo crea lazos afectivos; es posible construir la paz desde escenarios donde se fraguó el conflicto, la violencia y la guerra (Tovar \& Sacipa, 2012). En la Tabla 1 se presenta la inherencia de los conflictos en todas las relaciones humanas, con dos caminos de solución: el violento y el pacífico. Se presenta la relación de cada uno de los tipos de violencia, con las manifestaciones dvve paz. De ahísurge la educación para la paz, que incluye la educación sobre paz (contenidos) y educación en paz (forma, metodología y ambientes de aula).

\section{UNA REVISION CUALITATIVA}

Desde un enfoque cualitativo se pretendió conocer por un lado la realidad de la Cátedra de la Paz de la Fundación ICPROC y, por otro lado, el pensamiento de Camilo Torres Restrepo. Respecto a la realidad de la Cátedra de la Paz, se tuvo en cuenta la opinión de los asesores pedagógicos de la Fundación, junto a cuatro de los docentes-tutores (uno por cada zona de trabajo); así mismo, el PEI de la Fundación ICPROC. Para el pensamiento de Camilo Torres Restrepo, se toman cuatro escritos, todos con alguna referencia a la educación: "Mensaje a los estudiantes",

\begin{tabular}{|c|c|c|c|}
\hline \multicolumn{2}{|c|}{$\begin{array}{l}\text { CONFLICTO } \\
\begin{array}{l}\text { Natural e inherente al hombre en sus relaciones sociales. Las soluciones se pueden dar } \\
\text { de dos maneras: }\end{array}\end{array}$} & \multirow{4}{*}{$\begin{array}{l}\text { E } \\
\text { D } \\
\text { U } \\
\text { C } \\
\text { A } \\
\text { C } \\
1 \\
0 \\
N\end{array}$} & \multirow{3}{*}{$\begin{array}{l}\text { Educación sobre paz } \\
\text { (se refiere a los contenidos) }\end{array}$} \\
\hline Violenta & Pacífica & & \\
\hline Violencia directa (actos concretos de violencia) & Paz negativa & & \\
\hline Violencia estructural (ejercida por el medio) & Paz positiva & & \\
\hline Violencia cultural (donde la cultura legitima la violencia) & & $\begin{array}{l}\mathbf{A} \\
\mathbf{R} \\
\mathbf{A}\end{array}$ & $\begin{array}{l}\text { Educación en paz } \\
\text { (forma, metodologías, ambientes } \\
\text { de aula) }\end{array}$ \\
\hline Violencia simbólica (asegura la dominación) & Paz neutra & $\begin{array}{l}\text { L } \\
\text { A } \\
\text { P } \\
\text { A } \\
\text { Z }\end{array}$ & \\
\hline
\end{tabular}

Tabla 1. Resolución de conflictos y Educación para la Paz. Fuente: Elaboración propia 
"Los problemas sociales en la Universidad actual", "La Universidad y el cambio social" y "Un sacerdote en la Universidad".

Para el desarrollo de la investigación, se tomaron tres instrumentos para la recolección de datos. El primero fue la entrevista semiestructurada, desde donde se abordó a los asesores pedagógicos y los docentes tutores de la Fundación ICPROC. Para la entrevista se plantearon inicialmente tres preguntas generales (sobre educación para la paz, sin abordar ninguna de las categorías) y 16 preguntas específicas (sobre cada uno de los indicadoresdecategoríaquesetomaroncomobaseparala educación para la paz en esta investigación), teniendo en cuenta cada uno de los indicadores de categoría: dignidad humana, bien común, solidaridad, cooperación, liderazgo, espiritualidad, forma, metodología y ambientes de aula. Al realizar la prueba piloto para la validación del instrumento se descubrió la necesidad de una nueva pregunta general, para un total decuatroyuna pregunta más para los primerosseis temas específicos, de manera que se ajustó a un total de 22 preguntas específicas.

El segundo instrumento para la recolección de datos fue el análisis semiótico del discurso, tomado de una análisis realizado previamente por el doctor Roberto Sancho Larrañaga (director del proyecto). Este instrumento nos permite tomar aspectos literales de las obras de Camilo Torres, que son el significante denotativo, que confrontado con el Tesauro de la UNESCO, nos arroja una categoría (significado); esta categoría se toma como significante connotativo, delcualsurgeunsignificado(valor). Elanálisis semiótico del discurso, nos conduce en lo denotativo a un código cultural, en lo connotativo a un mito. Como último instrumento utilizamos el análisis de documento, con el cual, a partir de una rúbrica, se analizaron tanto el PEI de la Fundación ICPROC, como la Ley 1732 de 2014 y el Decreto 1038 de 2015, por los cuales se implementa y reglamenta la Cátedra de la Paz en todas las instituciones educativas del país.

El análisis de los datos se realiza a partir de las categorías y los indicadores de categoría que se definieron según el marco teórico. La educación para la paz, comprende dos grandes categorías, educación sobre paz y educación en paz. La educación sobre paz, se refiere a los contenidos, los autores coinciden en señalar que deben ser valores, en la multiplicidad de los presentados, se eligieron para la investigación seis valores:dignidad humana, bien común, solidaridad, cooperación, liderazgo y espiritualidad. La educación en paz, se aborda desde tres indicadores de categoría: forma, metodología y ambientes de aula.

El análisis de entrevista se realizó desde una rúbrica, en la que se tomó las respuestas de cada uno de los entrevistados, por cada indicador de categoría de la investigación, de la respuesta de cada entrevistado, se resaltaba una idea fuerza, esta idea se confrontaba con el Tesauro de la UNESCO en Educación, lo cual nos permitía encontrar una categoría. Por otra parte, el análisis semiótico del discurso, se realizó, según lo indicado en un párrafo anterior. Finalmente, la rúbrica del análisis de documento, nos arrojó un calificativo según el nivel de uso de cada uno de los indicadores de categoría, en los documentos sometidos a dicho análisis.

Lo anteriormente expuesto, nos condujo a organizar los resultados de la manera que describimos a continuación. Se tomaron los indicadores de categoría uno a uno, para presentar los resultados de las entrevistas, 
del análisis semiótico del discurso y del análisis de documento. En el análisis de entrevista se presentó el conjunto de respuestas de cada una de las preguntas, que nos conducía a una conclusión del indicador de categoría. De la misma manera se analizó el indicador de categoría en cada uno de los cuatro documentos de Camilo Torres Restrepo, que nos llevaba a una conclusión, desde Camilo, de cada indicador. Así mismo, cada indicador fue analizado en los tres documentos propuestos. Se conjugaron las conclusiones para llegar a una conclusión general de la categoría, es decir una conclusión de educación sobre paz y una conclusión de educación en paz.

\section{ALGUNOS RESULTADOS}

En los hallazgos que pudimos obtener de la investigación encontramos a nivel general que la Cátedra de la Paz debe ser tratada comoun proyecto transversal y centrarse en la persona humana como ser en relación. Particularmente, en la categoría de educación sobre paz, se pudieron establecer las perspectivas y metodologías, para cada uno de los valores a tratar en los ciclos lectivos especiales integrados de la Fundación ICPROC. En la categoría de educación en paz, se logró identificar la perspectiva y metodología, desde la que se puede crear un contexto más pacífico dentro de la misma Fundación.

Respecto a la transversalidad de la Cátedra de la Paz, no encontramos autores que la contradijeran, en cambio sí, autores que corroboraron esta premisa, tales como Vidanes Díez (2007), Belmonte y otros (1990), Olleros González (2000), quienes consideran improcedente tratarla como una disciplina independiente, ya que ella toca todas las realidades de la Escuela. En referencia a la centralidad de la persona en la Cátedra de la Paz, aparecen autores como Gómez Arévalo (2015),
Zurbano Díaz de Cerio (1998) y Cerdas (2013), quienes presentan toda educación para la paz, como educación del ser humano, para la vida y para solucionar los conflictos que se presentan en sus relaciones. A lo largo de la investigación, no se encontraron autores que contradijeran esta postura.

En cuanto a las perspectivas y metodologías, que se encontraron para las dos categorías de análisis concluimos: la educación en paz se propone desde una perspectiva política y existencial, con metodología ver, juzgar, actuar. La educación sobre paz, aparece desarrollada en cada uno de los valores propuestos. Dignidad humana desde una perspectiva existencial con cualquier metodología que propicie la interrelación, bien común desde una perspectiva social y económica con cualquier metodología que propicie la interrelación, solidaridad desde una perspectiva social y económica con meto dología de aprendizaje activo, cooperación desde una perspectiva económica con metodología de talleres, liderazgo desde una perspectiva política con metodología de desarrollo de las habilidades, y espiritualidad desde una perspectiva religiosa y existencial con metodología de seminario.

Ahora bien como el objetivo de la investigación fue abrir espacio a nuevos estudios se propuso la posibilidad de aplicar los lineamientos en una Cátedra de la Paz, con contenidos y estrategias dentro de la Fundación ICPROC, una nueva investigación de la Catedra de la Paz, desde una mirada distinta a la de Camilo Torres Restrepo, o como nuevo interrogante, Camilo ¿cura o guerrillero?

\section{REFERENCIAS}

Alvarado, S. V., Opina, H. F., \& Luna, M. T. (2006). Transformación de actitudes frente a la equidad en 
niños y niñas de sectores de alta conflictividad social, en un proceso de socialización política y educación para la paz. Revista Latinoamericana de Ciencias Sociales, niñez y juventud, 1-26.

Añorve, M. A. (1991). La fiabilidad en la entrevista: la entrevista semiestructurada y estructurada, un recurso de la encuesta. Portal de revistas científicas y arbitradas de la UNAM, 29-37.

Arellano, N. (2007). La violencia escolar y la prevención del conflicto. ORBIS/ciencias humanas, 23-45. Ballón, E. (1997). Análisis semiótico del discurso. Madrid: G redos.

BBC Mundo. (20 de septiembre de 2015). BBC Mundo. Recuperado el 15 de noviembre de 2015, de http://www.bbc.com/mundo/notcias/2015/09/150920 _papa_francisco_cuba_homilia_misa_habana_revolucion_ac

Belmonte, P., Carrasco, A., \& Lario, M. (1990). Educación para la paz: algunas vías de investigación y reflexión desde el movimiento pacifista. Pedagogía social: revista interuniversitaria, 191-203.

Caballero, M. J. (2010). Convivencia escolar. Un estudio sobre buenas prácticas. Paz y Conflictos, 154-169.

Calvo, G. (2003). La escuela y la formación de competencias sociales: un camino para la paz. Educación y Educadores, 69-90.

Campoy, T., \& Gomes, E. (2009). Técnicas e instrumentos cualitativos de recogida de datos. En A. Pantoja Vallejo, Manuel básico para la realización de tesinas, tesis y trabajos de investigación (págs. 277-304). Madrid: EOS.
Cerdas, E. (2013). Educación para la Paz: Fundamentos teóricos, epistemológicos y axiológicos. Revista Latinoamericana de Derechos Humanos, 189-201.

Charria, A. (10 de Febrero de 2016). EL ESPECTADOR. Obtenido de http://www.elespectador.com/opinion/ catedra-de-paz-0

Clausó, A. (1993). Análisis documental: el análisis formal. Revista general de Información y Documentación, 11-19.

conocimientosWeb.net. (24 de Enero de 2017). CONOCIMIENTOSWEB.NET. Obtenido de http://www. conocimientosweb.net/portal/article292.html

Dulzaides, M. E., \& Molina, A. M. (marzo-abril de 2004). ACIMED. Scielo. Obtenido de Scielo:http:// scielo.sld.cu/scielo.php?script $=$ sci_arttext\&pid=S1024-94352004000200011

El nuevo Siglo. (27 de Enero de 2017). EL NUEVO SIGLO. Obtenido de EL NUEVO SIGLO: http://www.elnuevosiglo.com.co/articulos/01-2017-farc-y-eln-procesos-amarchas-muy-forzadas

ELTIEMPO.COM. (24 de Noviembre de 2016). EL TIEMPO. Obtenido de http://www.eltiempo.com/politica/ proceso-de-paz/ceremonia-de-la-firma-del-nuevo-acuerdo-de-paz-en-vivo/16757839

Fernández, A. (2000). Educación para la paz. La educación hoy. Concepto, interrogantes y valores, 211-229. Fisas, V. (2006). Cultura de paz y gestión de conflictos. España: Ediciones gráficas Rey S.L.

Fisas, V. (2011). Educar para una Cultura de Paz. Quaderns de construcció de pau, 1-10. 
Fontanille, J. (09 de Febrero de 2008). Semiótica de los textos y de los discursos. SEMIO. UIS. Obtenido de SEMIO. UIS: http://semiouis.blogspot.com.co/2008/02/ semitica-de-los-textos-y-de-los.html\#

Freire, P. (1997). La Educación como práctica de la libertad. México: Siglo veintiuno editores.

Fundación Cultura de Paz. (04 de Junio de 2016). Fundación Cultura de Paz. Obtenido de www.fund-culturadepaz.org/spa/principal/Cultura\%20Paz.htm Fundación para la Aplicación y Enseñanza de las Ciencias. (01 de Junio de 2016). Sistema de Aprendizaje Tutorial. Fundaec. Obtenido de www.fudaec.org/es/programas/sat/index.htm

Fundación Pro-Cultura. Comité de Solidaridad con los Presos Políticos. Revista Solidaridad. Movimiento de Cristianos por el Socialismo. (1985). CAMILO TORRES RESTREPO. Escritos. Bogotá.

García, L., \& López, R. (2011). Convivir en la escuela. Una propuesta para su aprendizaje por competencias. Educación, 531-555.

Giraldo, P. J. (2015). Camilo "regresa". En O. d. sociales, Camilo, ayer y hoy, signo de reconciliación (pág. 170). Santiago de Cali: Merlín SAS.

Gómez, A. P. (2012). Educación para la paz en el sistema educativo de El Salvador. Ra Xhimai, 93-126.

Gómez, A. P. (2015). Una apuesta educativa para América Latina: educación para la paz. Revista Latinoamericana de Estudios Educativos, 17-62.

Gómez, M. E. (2012). La tutoría académica en la facultad de Ciencias políticas y sociales de la Universidad Autónoma de México desde la perspectiva de educación para la paz. México: Miguel Ángel Porrúa.
Hirmas, C., \& Carranza, G. (2008). Matriz de indicadores sobre convivencia democrática y cultura de paz en la escuela. Educación para la paz, la convivencia democrática y los derechos humanos (págs. 56-136). San José de Costa Rica: Salesianos impresores S.A.

Houtart, F. (2015). Camilo Torres Restrepo y el Proceso de Paz de Colombia. En Observatorio de Realidades Sociales, Camilo, ayer y hoy, signo de reconciliación (pág. 170). Santiago de Cali: Merlín SAS.

ICPROC. (2013). Proyecto Educativo Institucional. San Vicente de Chucurí.

ICPROC. (30 de Mayo de 2016). Fundación Instituto Cristiano de Promoción Campesina. Obtenido de http:// www.icproc.org.co/script/asisomos/index.php?idi=1

Jiménez, F. (2012). Conocer para comprender la violencia: origen, causas y realidad. Convergencia, 13-52.

Jiménez, M., Lleras, J., \& Nieto, A. M. (2010). La paz nace en las aulas: evaluación del programa de reducción de la violencia en Colombia. Educación y Educadores, 347-359.

Juan XIII, P. (1963). Pacem in Terris. Ciudad del Vaticano: Paulinas.

Juanola, E. (2012). Perdón y reconciliación para vivir en paz. En E. Vinyamata, M. Albaladejo, I. Armadans, M. Burguet, E. Castellano, P. Horno. R. Urrea, Vivir y convivir en paz. Aprender a vivir con uno mismo y con el otro, 70-82. Barcelona: GRAÓ.

López, M. d., \& Fernández, A. (2014). Educar para la paz. Necesidad de un cambio epistemológico. Convergencia. Revista de ciencias sociales, 177-142. 
López, R. E., \& Deslauriers, J. P. (2001). La entrevista cualitativa como técnica para la investigación en Trabajo Social. Margen, 1-19.

Martín, C. (07 de abril de 2009). Temas de Biblioteconomía. e-LiS. Obtenido de e-LiS: http:// eprints.rclis.org/14605/1/tipdoc.pdf

Martínez, M. (2006). La investigación cualitativa (síntesis conceptual). Revista de investigación en Psicología, 123-146.

Martínez, J. (2011). Métodos de investigación cualitativa. Silogismo, 1-43.

Mayan, M. (2001). Una introducción a los métodos cualitativos: Módulo de entrenamiento para estudiantes y profesionales. México: Qual Institute Press.

Monsalve, M. D. (2015). Reintegrar a Camilo. En Observatorio de Realidades Sociales, Camilo, ayer y hoy, signo de reconciliación (pág. 170). Santiago de Cali: Merlín SAS.

Moriano, J. A., Molero, F., \& Lévy, J. P. (2011). Liderazgo auténtico. Concepto y validación del cuestionario ALQ en España. Psicothema, 336-341.

Murillo, J., \& Hernández, R. (2011). Hacia un concepto de justicia social. Revista Iberoamericana sobre Calidad, Eficacia y Cambio en Educación, 7-23.

Olleros, B. (2000). Educación para la paz. Contextos educativos, 158-162.

Ortega, R. (2007). La convivencia: un regalo de la cultura a la escuela. Idea La Macha, 50-54.
Oswald, ú. (2001). Estudios para la paz desde una perspectiva global: necesidades humanas en un mundo interrelacionado. México: Miguel Ángel Purrúa.

Pérez, J. (20 de Enero de 2007). Estudio exploratorio sobre el tema de la espiritualidad en el ambiente laboral. Anales de psicología, 137-146. Obtenido de DIGITUM Biblioteca Universitaria: http://hdl.handle.

net/10201/8114

Pérez, O. (2012). Algunos porqués cognitivos del análisis semiótico: Una aproximación a las confluencias entre Semiótica y Psicología Cognitiva. ZER, 101-117. Pontificio Consejo "justicia y Paz". (2005). Compendio de la Doctrina Social de la Iglesia. Ciudad del Vaticano: Paulinas.

Pontificio Consejo "vida y paz". (2005). Compendio de la Doctrina Social de la Iglesia. Ciudad del Vaticano: Paulinas.

Real Academia Española. (20 de Enero de 2017). Diccionario de la Lengua Española. Obtenido de http:// dle.rae.es/?id=Aid2o2x

Salamanca, M., Rodríguez, M., Ovalle, R., Cruz, J. D., Pulido Albarracín, M. Á., \& Molano Rojas, A. (2016).

Guía para la implementación de la Cátedra de la Paz. Bogotá: SANTILLANA S.A.S.

Salamanca, A. P. (2009). Educación para la paz. Praxis, 1-16.

Salazar, I. I., \& Sandoval, E. A. (2009). Paz y ciudadanía en el proceso de formación básica de los jóvenes de secundaria. México: Universidad de México. 
Sánchez, S. L. (2007). Análisis semiótico discursivo de las representaciones sociales de la juventud difundidas en los Mass Media. Mediaciones, 13-25.

Santander, P. (2011). Por qué y cómo hacer análisis de discurso. Cinta Moebio, 207-224.

Terra. (19 de Enero de 2016). Terra. Obtenido de https://noticias.terra.com.co/camilo-torres-una-figura-incomoda-para-la-derecha-y-la-izquierda,8bf33c81196078602214c4e076cc8b3bk5huotes.html

Torres, C. (1986). La violencia y los cambios socio-culturales en las áreas rurales colombianas. En Camilo Torres Restrepo escritos escogidos (pág. 379). Bogotá: Cimarrón Editores.

Tovar, C. C., \& Sacipa, S. (2012). Significados e interacciones de paz de jóvenes integrantes del grupo Juventud Activa de Soacha, Colombia. Universitas Psychologica, 35-46.

Umaña, E. (1986). CAMILO TORRES RESTREPO. Escritos escogidos. Bogotá: CIMARRÓN EDITORES.

Umaña, E. (1986). Vigencia de Camilo. En Camilo Torres Restrepo Escritos escogidos (pág. 379). Bogotá: Cimarrón Editores.

UNESCO. (04 de Junio de 2016). Proyecto Transdisciplinario "Hacia una cultura de paz". Obtenido de www.unesco.org/cpp/sp/proyectos/cppinfo.htm

UNESCO. (22 de Enero de 2017). Tesauro de la UNESCO. Obtenido de Tesauro de la UNESCO: http:// vocabularies.unesco.org/browser/thesaurus/es/page/ concept1482

Valencia, Á. (2009). Mis adversarios guerrilleros. Bogotá: Planeta Colombiana S.A.
Valencia, Á. (2009). Mis adversarios guerrilleros. Bogotá: Planeta Colombiana S.A.

Valencia, R. (2015). La implantación de educación para la ciudadanía en el sistema educativo español. Madrid: Dykinson.

Vidanes, J. (2007). La educación para la paz y la no violencia. Revista iberoamericana de Educación.

Zepeda, R. (2004). El espacio político en que se construye la cultura de paz en Guatemala: investigación sobre los aspectos sociopolíticos que la permiten o la obstaculizan. Guatemala: FLACSO.

Zurbano, J. L. (1998). Bases de una educación para la paz y la convivencia. Pamplona: Gobierno de Navarra. Departamento de Educación y Cultura.

Citar este artículo como: Carrillo, R. (2017). "Lineamientos para la cátedra de la paz en la Fundación Instituto Cristiano de Promoción Campesina (ICPROC), a partir del pensamiento de Camilo Torres Restrepo". En: Revista La Tercera Orilla (19). Bucaramanga: Universidad Autónoma de Bucaramanga. 
LA TERCERA ORILLA\#19, Diciembre 2017

69 